OPEN ACCESS

Edited by:

Mikhail Lebedev,

Duke University, United States

Reviewed by:

Dimiter Dimitrov

Diavita Ltd., Bulgaria

Marta Parazzini,

Consiglio Nazionale Delle Ricerche

(CNR), Italy

*Correspondence:

Elena Ratti

elena.ratti@biogen.com

Received: 27 April 2017

Accepted: 18 July 2017

Published: 03 August 2017

Citation:

Ratti E, Waninger S, Berka C, Ruffini G and Verma $A$ (2017) Comparison of Medical and Consumer Wireless EEG

Systems for Use in Clinical Trials.

Front. Hum. Neurosci. 11:398.

doi: 10.3389/fnhum.2017.00398

\section{Comparison of Medical and Consumer Wireless EEG Systems for Use in Clinical Trials}

\author{
Elena Ratti ${ }^{*}$, Shani Waninger ${ }^{2}$, Chris Berka ${ }^{2}$, Giulio Ruffini ${ }^{3}$ and Ajay Verma ${ }^{1}$ \\ ${ }^{1}$ Biogen, Cambridge, MA, United States, ${ }^{2}$ Advanced Brain Monitoring, Inc., Carlsbad, CA, United States, ${ }^{3}$ Neuroelectrics \\ Corporation, Cambridge, MA, United States
}

Objectives: To compare quantitative EEG signal and test-retest reliability of medical grade and consumer EEG systems.

Methods: Resting state EEG was acquired by two medical grade (B-Alert, Enobio) and two consumer (Muse, Mindwave) EEG systems in five healthy subjects during two study visits. EEG patterns, power spectral densities (PSDs) and test/retest reliability in eyes closed and eyes open conditions were compared across the four systems, focusing on Fp1, the only common electrode. Fp1 PSDs were obtained using Welch's modified periodogram method and averaged for the five subjects for each visit. The test/retest results were calculated as a ratio of Visit 1/Visit 2 Fp1 channel PSD at each $1 \mathrm{~s}$ epoch.

Results: B-Alert, Enobio, and Mindwave Fp1 power spectra were similar. Muse showed a broadband increase in power spectra and the highest relative variation across test-retest acquisitions. Consumer systems were more prone to artifact due to eye blinks and muscle movement in the frontal region.

Conclusions: EEG data can be successfully collected from all four systems tested. Although there was slightly more time required for application, medical systems offer clear advantages in data quality, reliability, and depth of analysis over the consumer systems.

Significance: This evaluation provides evidence for informed selection of EEG systemsappropriate for clinical trials.

\footnotetext{
Keywords: neurophysiology, electrophysiology, electroencephalogram, quantitative EEG, clinical trials, consumer EEG
}

\section{INTRODUCTION}

Neurodegenerative disorders represent huge global unmet medical needs and require the development of new disease modifying therapies. Given the insidious nature of these disorders and the high cost of many diagnostic tests, there is a significant need for widely available, reliable, and inexpensive biomarkers to track progression of neurodegenerative processes in time frames suitable for drug development. In this context, EEG may have remarkable potential. Although EEG is susceptible to known lifestyle factors and medications, it has many considerable advantages. EEG reflects synaptic activity, which is a common denominator for the functional impact of neurodegenerative processes. EEG is a non-invasive, portable, safe, and inexpensive technology that is widely accepted and requires relatively short acquisition time. Qualitative EEG is routinely utilized in clinical practice for the diagnosis of epilepsy. More recently, an integration 
of a quantitative EEG biomarker (qEEG) and clinician's evaluation have been proposed for the assessment of attentiondeficit/hyperactivity disorder (ADHD) and has been granted from the FDA (Food and Drug Administration) class II designation to support the clinical evaluation of ADHD (Lenartowicz and Loo, 2014; Snyder et al., 2015). qEEG is in investigational stages for use as an endpoint in neurodegenerative diseases in clinical trials. However, recent advances in data analyses, interpretation and improved spatial resolution have increased the potential of EEG as a reliable, accurate biomarker for neurodegenerative disease progression. Many reported observational resting state qEEG analyses support its potential value as a biomarker for detection of neural signatures of neurodegeneration occurring in Alzheimer's disease (Babiloni et al., 2011; Moretti et al., 2011; Berka et al., 2014; Chen et al., 2015; Garn et al., 2015; Ruffini et al., 2016; Waninger et al., 2016), Parkinson's disease (Sarnthein and Jeanmonod, 2007; Babiloni et al., 2011; Soria-Frisch et al., 2014; Shani Waninger et al., 2015; Kroupi et al., 2017) and frontotemporal dementia (Pijnenburg et al., 2008; Nishida et al., 2011; Caso et al., 2012; Goossens et al., 2016).

Over the years, EEG hardware technology has also evolved and several wireless multi-channel systems have emerged that deliver high quality EEG and physiological signals in a simpler, more convenient and comfortable design than the traditional, cumbersome systems. Traditional EEG systems require lengthy assembly and application time, typically involving abrasion of the patient's scalp. The application time and discomfort render these traditional systems challenging to use in populations affected by dementia, where cooperation with lengthy clinical procedures is often difficult. However, several currently available wireless systems can be applied in $20 \mathrm{~min}$ or less with no discomfort during application and with a comfortable fit during acquisitions. Combined with advances in signal detection and quantitative analysis techniques, wireless systems are ideal candidates for relatively rapid, tolerable clinical assessment of potentially challenging dementia populations, such as behavioral variant frontotemporal dementia, characterized by prominent behavioral and personality changes.

More recently, there has also been a growing market for consumer wearable technologies leading to limited-channel systems available for personal use, such as meditation and relaxation training. It is conceivable that these systems, albeit their limited coverage, may also be used in selected clinical studies. Application of these consumer systems in clinical trials research has not been extensively explored however and the accuracy and reliability of these systems for repeated measurements have not been well-established. Further, it is not clear whether the limited-channel acquisition may provide sufficient data and anatomical coverage to assess the neural signatures in patients affected by neurodegenerative diseases.

The current study was designed to provide initial evaluation of the potential of consumer EEG systems for clinical trials, by comparing the ease of use, accuracy and reliability of two medical grade, multi-channel wireless EEG systems, B-Alert X24, and Enobio 20, with two consumer, limited-channel systems, Muse and Mindwave.

\section{MATERIALS AND METHODS}

\section{Participants}

The study population included five healthy participants who met eligibility for the study (Table 1). Subjects were excluded if, after review of their medical history, concomitant medications, and lifestyle (alcohol and caffeine consumption as well as smoking status), they were not considered healthy. Specifically, they were excluded from the study if they had a history of epilepsy, or other sleep, neurological or psychiatric disorders or were taking medications or had a lifestyle known to affect EEG signal such as smoking and considerable alcohol and caffeine consumption.

Written informed consent was obtained from all the study participants following the guidelines for experimental investigation with human subjects required by the Chesapeake Institutional Review Board.

\section{Study Procedures, EEG Data Acquisition, and Analysis}

To avoid potential EEG signal variability associated with nutritional intake and circadian variations, all visits occurred in the morning following a standard low carbohydrate, high protein breakfast (to minimize post-prandial drowsiness) at the Advanced Brain Monitoring (ABM) research labs study site. In addition, participants were asked to avoid alcoholic beverages the night before the study visit as well as to fast and avoid caffeine on the morning of the visit.

EEG data was acquired during two separate visits $\sim 1$ week apart from two multi-channel (20 channels) medical EEG systems, B-Alert (Advanced Brain Monitoring, ABM) and Enobio (Neuroelectrics) (Figure 1, Table 2) and two limitedchannel consumer systems, Muse (Interaxon, 2 channels) and Mindwave (Neurosky, one channel) (Figure 1, Table 2) in the following order: Muse, Mindwave, B-Alert, Enobio. The B-Alert X24 EEG System and Enobio are both CE medically certified 20 channel wireless systems applied in the standard international 10-20 montage and acquire EEG signal at a sampling rate of 256 $\mathrm{Hz}$. In addition, B-Alert has been cleared by the FDA for use as a medical device.

B-Alert uses mastoids as a reference channel. Enobio can be used with a mastoid reference, ear-clip or using other scalp locations. Mindwave also has a reference on the ear slip and Muse has three reference channels on the forehead. All the systems included signal quality check, however as opposed to the medical EEG systems, both consumer systems have dry electrodes and no impedance check.

These technologies were selected based on their ability to provide end-users with raw EEG outputs through practical and non-cost-prohibitive access.

Ten minutes of resting state EEG was acquired during eyes open (EO) with visual fixation on a cross symbol presented on a computer screen ( $5 \mathrm{~min}$ ) as well as while eyes closed (EC) for 5 $\min$.

Since all four systems only share the FP1 channel (Figure 1), EEG patterns (raw and decontaminated), power spectral densities (PSDs), and test/retest reliability comparisons across the four 
TABLE 1 | Study population demographics and baseline characteristics.

\begin{tabular}{|c|c|c|c|c|c|c|}
\hline & Subject 1 & Subject 2 & Subject 3 & Subject 4 & Subject 5 & Group measures $^{a}$ \\
\hline Age (years) & 25 & 23 & 27 & 37 & 23 & $27 \pm 5.8$ \\
\hline Gender (male) & Male & Male & Female & Male & Female & $60 \%$ \\
\hline Handedness (right) & Right & Right & Right & Right & Right & $100 \%$ \\
\hline Education (years) & $\begin{array}{l}17 \text { Undergraduate } \\
\text { Degree }\end{array}$ & $\begin{array}{l}17 \text { Undergraduate } \\
\text { degree }\end{array}$ & $\begin{array}{l}17 \text { Undergraduate } \\
\text { degree }\end{array}$ & $\begin{array}{l}19 \text { Advanced } \\
\text { Degree }\end{array}$ & 17 Undergraduate degree & $17.4 \pm 0.9$ \\
\hline $\begin{array}{l}\text { Caffeine consumption } \\
\text { (number of coffees/day) }\end{array}$ & $2-3$ & $1-2$ & 1 & $1-2$ & $0-1$ & $1.8 \pm 0.8$ \\
\hline $\begin{array}{l}\text { Alcohol consumption } \\
\text { (number of drinks/day) }\end{array}$ & 0 & 0 & 1 & $0-2$ & $0-1$ & $0.8 \pm 0.8$ \\
\hline Smoking & No & No & No & No & No & $0 \%$ \\
\hline
\end{tabular}

a Categorical variables: N (\%); Continuous variables: Mean \pm Standard Deviation (SD).

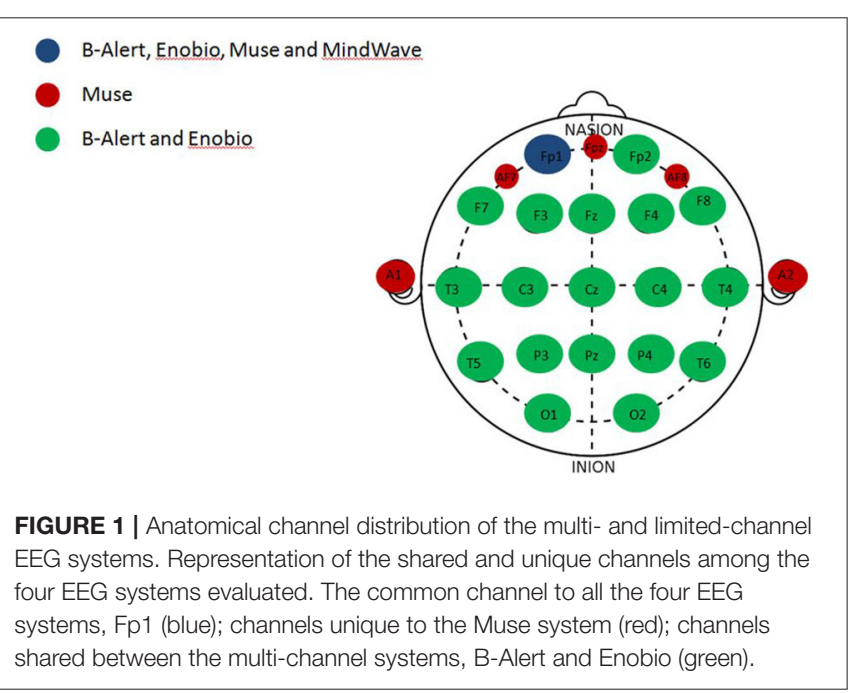

EEG systems evaluated in the study were performed focusing on the Fp1 electrode in both EO and EC conditions.

The EEG data from each system were loaded into MATLAB (Mathworks) using custom built functions. The EEG data recorded during breaks and instructions were discarded prior to analysis. Power spectral density of EEG for each $1 \mathrm{~s}$ epoch was calculated using Welch's modified periodogram method with a Hamming window tapering of $1 \mathrm{~s}$ length. PSDs were calculated on resting EEG during both eyes open and eyes closed periods. To allow equal units $(\mu \mathrm{V})$, a correction factor of 1.25 (Muse), 0.25 (MindWave), or 1,000 (Enobio 20) was applied. All Fp1 channel PSD data were averaged for the five subjects for each visit. Testretest was performed by calculating and plotting Vist1/Visit2.

\section{RESULTS}

\section{Participants}

Participants were all healthy volunteers with an average age of 27 years, they were all right handed and non-smokers, and predominantly males (60\%). Their demographics and baseline characteristics are summarized in Table $\mathbf{1}$.

\section{Resting State EEG}

EEG data was successfully collected from all four systems tested. As expected, due to the dry electrodes and the limited number of channels, the set up time was considerably less for the consumer EEG systems. However, there was no observed difference in subjects' tolerance and acceptance across the four systems.

Patterns of raw EEG data in EO (Figure 2) and EC (Figure 3) conditions were collected and evaluated from each system. Muse and Mindwave were more prone to artifact due to eye blinks and muscle movement in the frontal region with eye opening (Figure 2).

\section{Fp1 Channel Power Spectral Densities (PSDs) Comparison}

Average PSDs in the Fp1 channel common to all the four EEG systems for Visit 1 and Visit 2 were calculated and were plotted for both EO (Figures 4A,B) and EC (Figures 4C,D) conditions. In the EO condition, B-alert and Enobio spectra were approximately equal, while Mindwave was slightly increased but followed a similar curve. A broadband increase in power was observed for PSDs acquired with the Muse system. Similarly, in the EC condition (Figures 4C,D), B-Alert, Enobio, and Mindwave PSDs were similar however increased broadband power was observed for Muse spectra, which also appeared to have higher variation than the other systems. Peaks at $8-12 \mathrm{~Hz}$ (alpha band) were visible in the spectra acquired with the B-Alert, Enobio, and Mindwave systems at each visit. While there was a clear alpha peak for the Muse power spectra on Visit 2, no peak was observed in the Visit 1 spectra.

\section{Fp1 Channel Resting EEG Test/Retest Comparison}

In the EC condition, the power spectral ratio was between 0.975 and 1.025 for B-Alert, Enobio, and Mindwave (Figure 5A). The Muse system PSD ratios had more variation then the other three systems with ratios between 1.125 and 1.225. In the EO condition, there appeared to be slightly more variation for Enobio in the slow waves Delta $(1-3 \mathrm{~Hz})$, Theta $(3-7 \mathrm{~Hz})$, and slow alpha (8-10) and for B-Alert and Mindwave in the faster waves Beta $(13-30 \mathrm{~Hz})$ and Gamma $(25-40 \mathrm{~Hz})$. However, ratios were 
TABLE 2 | EEG systems comparisons.

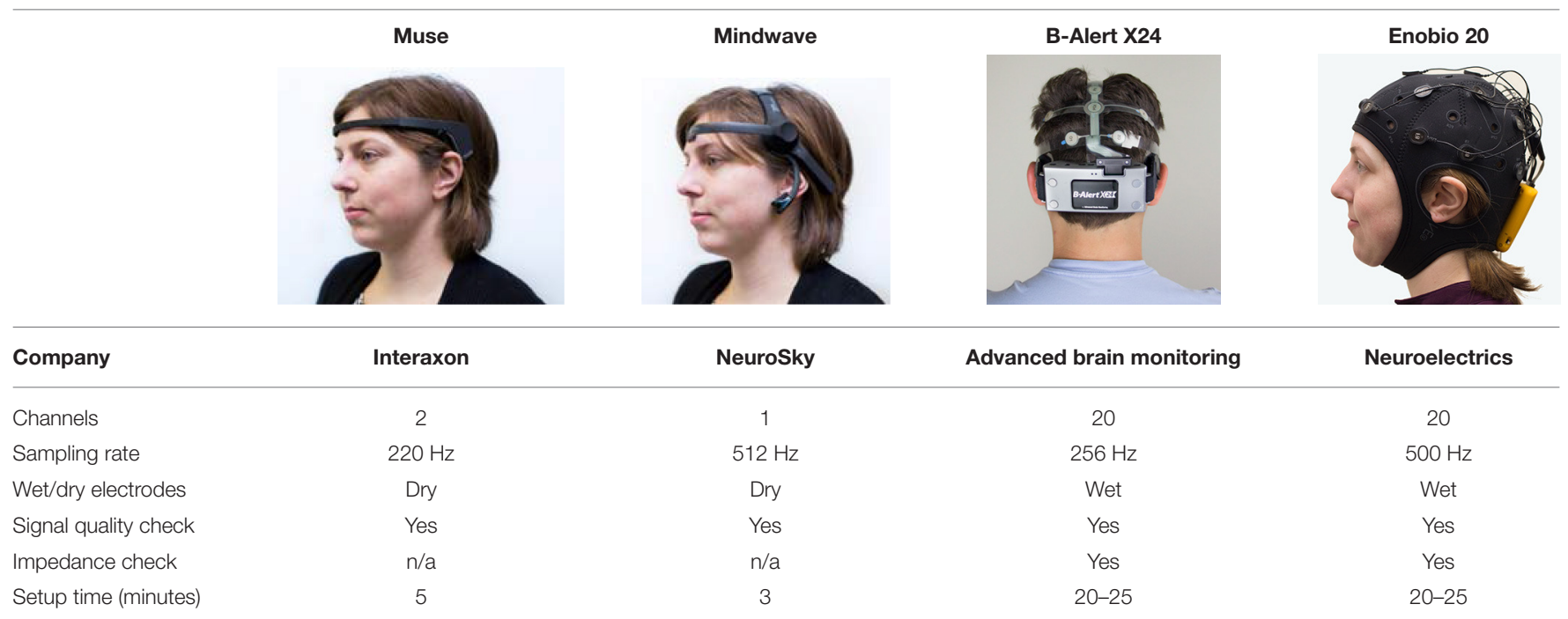

A

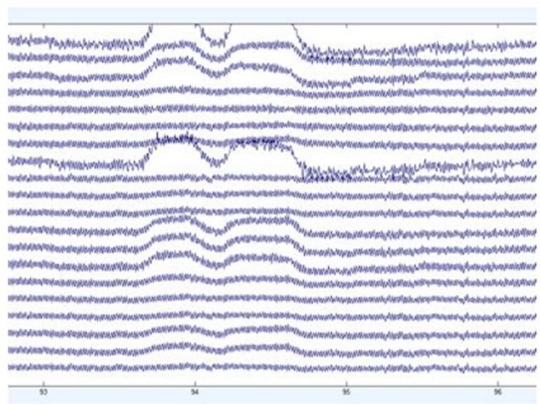

C

EO-Visit 2: S1056 B-Alert RAW

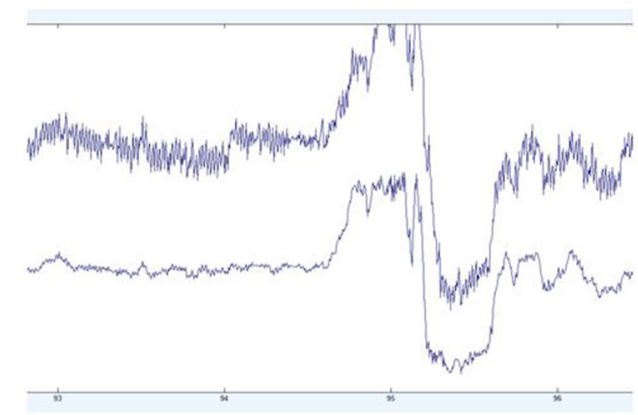

EO-Visit 2: S1056 Muse RAW
B

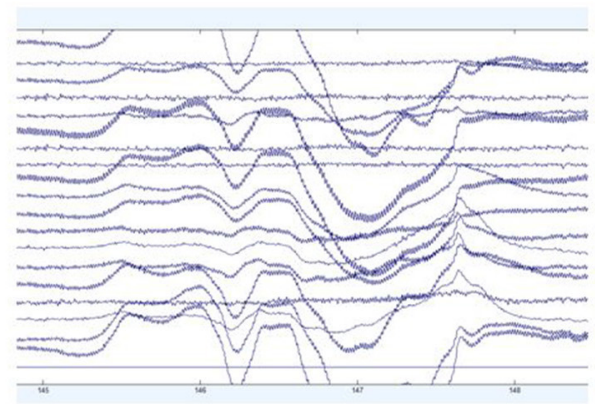

EO - Visit 2: S1056 Enobio RAW

D

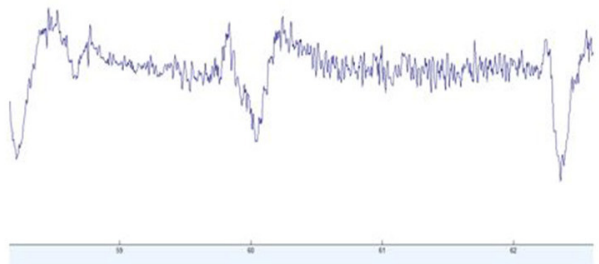

EO-Visit 2: S1056 MindWave RAW

FIGURE 2 | Extracts of EO raw data from B-Alert (A) and Enobio (B) Muse (C) and MindWave (D) within the same participant during the same visit.

still between 0.975 and 1.05. Similarly to the EC condition, the test/retest for Muse had higher variation, with ratios up to 1.2 (Figure 5B).

\section{DISCUSSION}

This study compared quantitative EEG signal and test-retest reliability of medical and consumer EEG systems in order to evaluate their potential application in clinical research and clinical trials. Newly popularized consumer EEG systems were evaluated due to their low cost, wide accessibility, and potential for home based studies in challenging populations. Among the popular applications of consumer EEG systems are meditation and relaxation training as well as coping with anxiety or pain. Recent investigations have been exploring their utility beyond gaming: NeuroSky was shown to be able to detect onset of stage 1 sleep (Van Hal et al., 2014) and there has also been interest 
A.

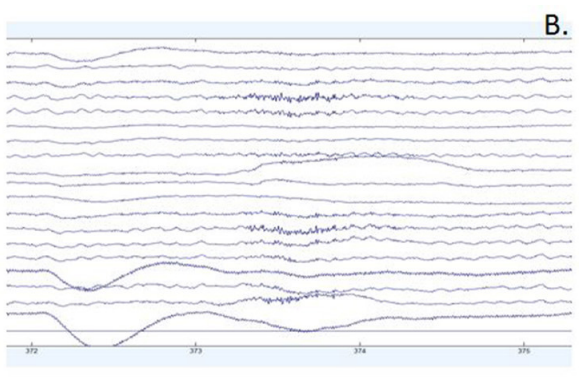

C.

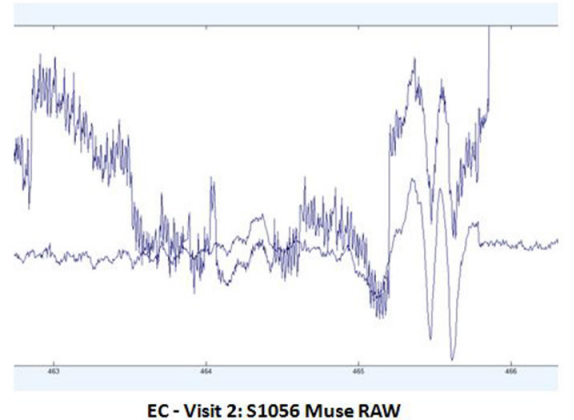

D.

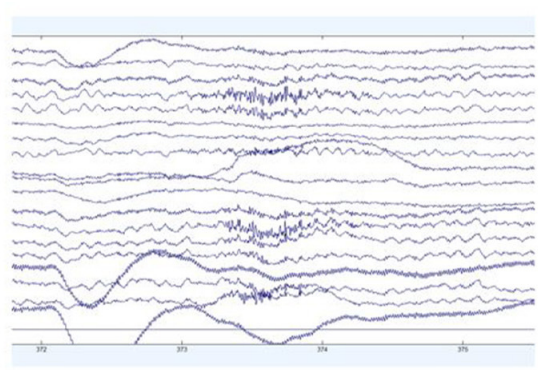

EC - Visit 2: S1056 Enobio RAW

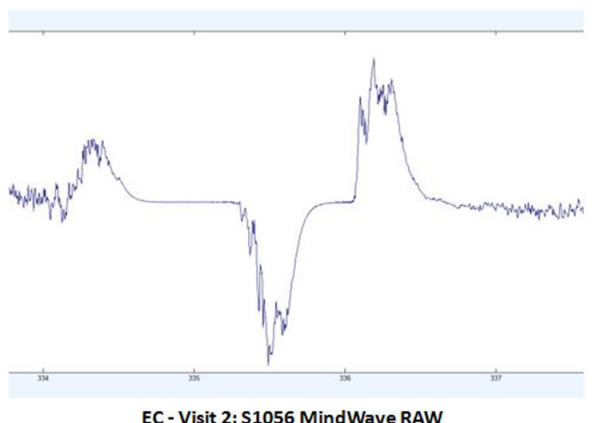

FIGURE 3 | Extracts of EC raw data from B-Alert (A) and Enobio (B) Muse (C) and MindWave (D) within the same participant during the same visit.

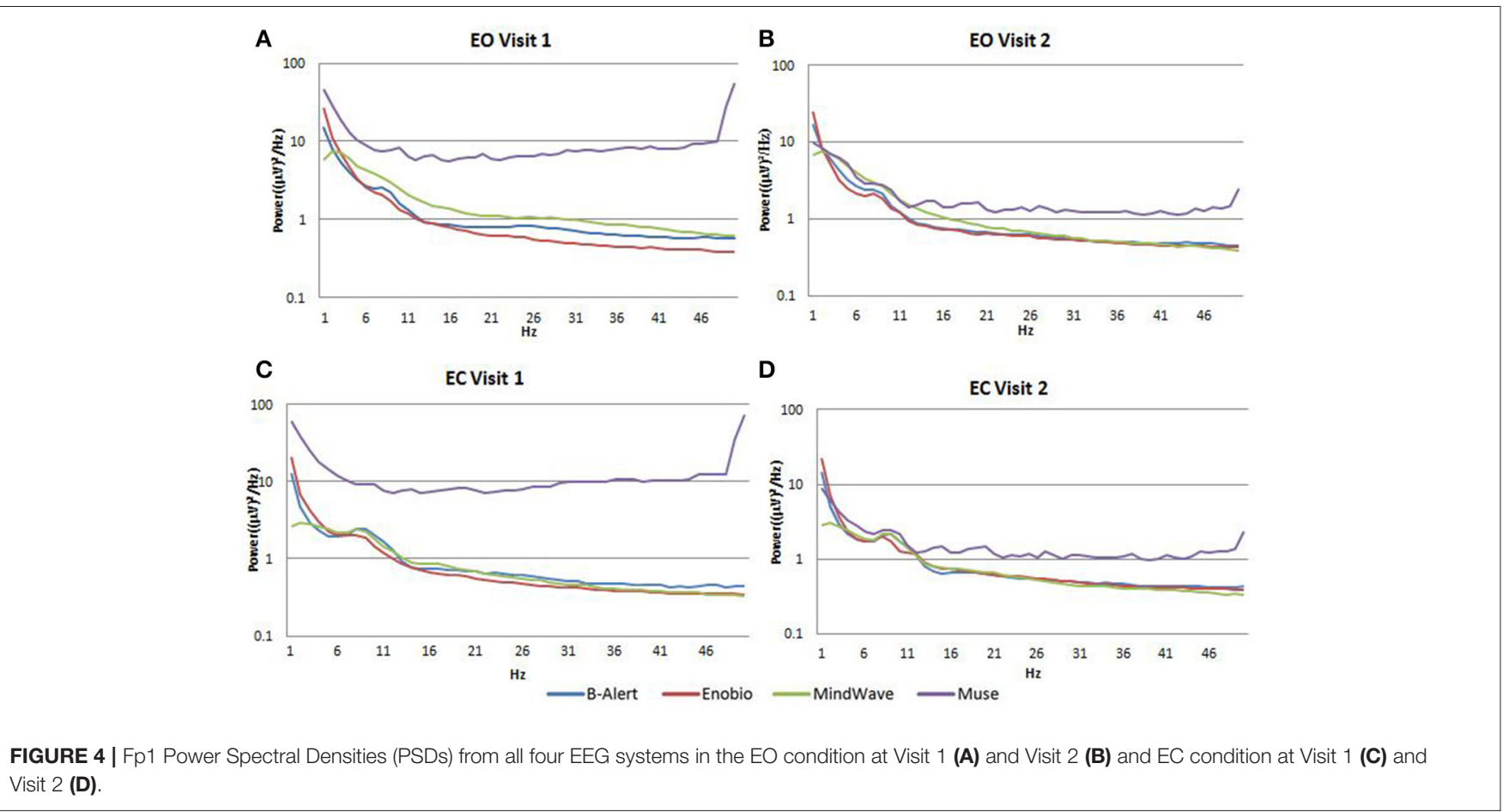

in assessing consumer EEG within brain computer-interfaces (Bialas and Milanowski, 2014; Kim et al., 2015; Taherian et al., 2017). The application of simpler EEG systems (6-channels, for example) is also being explored for emergency settings (Jakab et al., 2014). A prior evaluation, although utilizing a more complex headset, showed that commercially available multi-lead consumer EEG systems, such as the Emotiv EPOC 16-electrode cap, may also have value in evaluating clinical conditions (Schiff et al., 2016).

This study provided evidence that fairly good quality EEG data can be successfully collected from consumer EEGs. However, there were distinctions in the power increase, test retest, and 

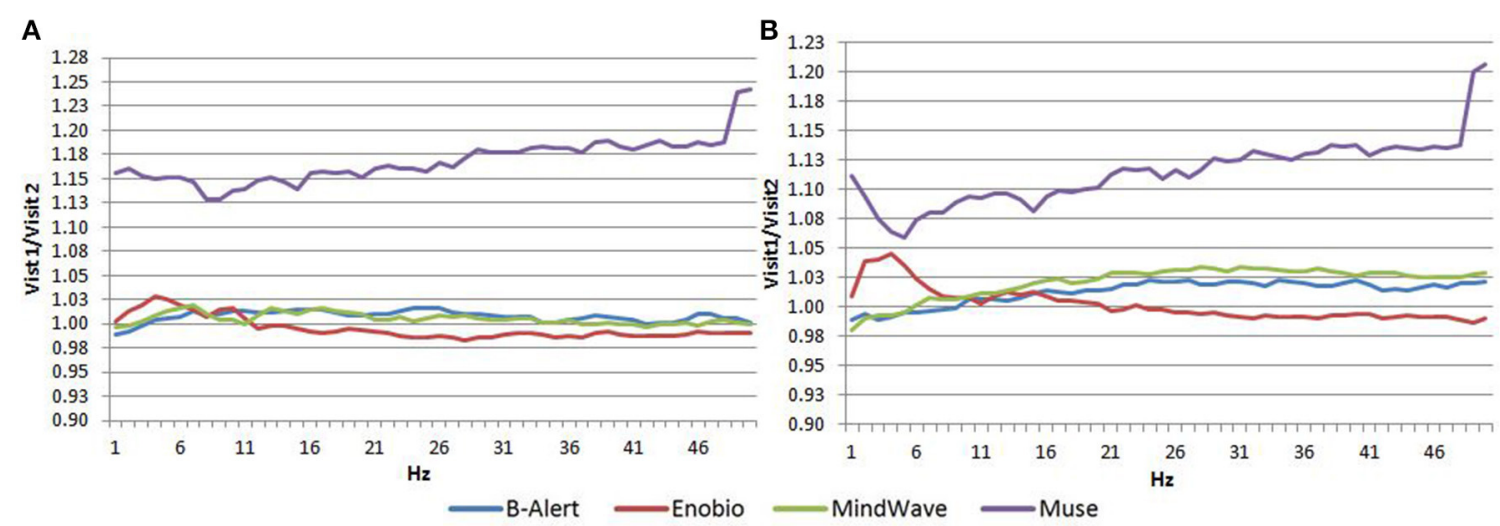

FIGURE 5 | Test-retest ratios for EC (A) and EO (B) condition.

shape of the alpha peak observed at 8-13 Hz. Mindwave showed overall similar Fp1 power spectra to the medical systems with a slight broadband increase over B-Alert and Enobio. Muse showed a broadband increase in power spectra, which may reflect artifact in the data acquired by a dry electrode.

Consumer EEG systems did show a significantly more convenient and faster set up,which is optimal for their intended use in entertainment and self-help applications. However, their data quality was overall negatively affected by artifact susceptibility associated with the dry electrode. As expected, the data quality was particularly diminished during EO. The lack of impedance testing capability and application to the frontal region, which is particularly prone to eye blinks and muscle movement with eye opening also likely contributed to this relative artifact. Additionally, the assessment performed by consumer EEG systems is, by their nature, limited and confined to the only anatomical brain region covered by the few channels, precluding multi-networks evaluations.

Dry electrodes may also be more prone to result in discomfort over time and pose a higher risk of misplacement on the forehead, leading to inaccurate signal acquisition and test/retest. Compared to medical grade equipment, test/retest reliability was lower in consumer EEG systems. Reliability was measured with a test-retest acquisition for all systems (Figure 5). While B-Alert, Enobio, and Mindwave performed reasonably well, Muse had relatively low reliability. Further, while a clear alpha peak was demonstrated for Muse on Visit 2 (Figure 4), the absence of an alpha peak on Visit 1 suggests a lack of consistency that may be due to artifact. Consistent reliable measures of brain activity are crucial in clinical trials when monitoring disease progression and evaluating efficacy of an experimental therapeutic. While the consumer systems may be useful for a quick assessment when time is limited, these limitations of consumer EEG could hinder their applications in research and clinical trials settings and a medical grade system with high test-retest reliability is recommended for use as a pharmacodynamic endpoint in clinical assessments.

Ultimately, the comparison of medical and consumer EEG systems under experimental conditions highlighted differentiation in performance and, particularly, specific limitations of use that could hinder the applications of consumer systems in research and clinical trials settings. On the other hand, the medical multi-lead systems are less ideal for entertainment purposes that require rapid setup and data processing from a minimal number of electrodes. The main limitation of this study relies in the small size of the study population and lack of randomization of order of systems used for acquisition. Data was acquired in the same order of systems in order to avoid effects of time of acquisition that can impact EEG due to circadian rhythms. However, EEG power metrics have proven to be a very reliable and repeatable for individuals when using standard EEG systems. For this reason, the selected sample size was considered informative.

In conclusion, EEG data can be successfully collected from all four systems tested, including consumer EEG systems, with varying limitations on usability, data quality and reliability that guide their optimal applications including in clinical trial settings. Susceptibility to artifact and variability in test/retest reliability associated with current consumer EEG systems suggest the use of medical grade EEG system for robust clinical cross-sectional and longitudinal EEG data collections.

\section{AUTHOR CONTRIBUTIONS}

ER and AV: participated in the conception and the design of the project, the analysis, and interpretation of data and manuscript preparation. SW, CB, and GR: participated in the conception and the design of the project, its execution, the analysis and interpretation of data and manuscript preparation. All authors have been involved in drafting, writing, and revising the manuscript and they all have read and approved the final version of the manuscript.

\section{FUNDING}

This study was supported by Biogen. 


\section{ACKNOWLEDGMENTS}

We would like to acknowledge the study participants for their time and contributions to this study. The

\section{REFERENCES}

Babiloni, C., De Pandis, M. F., Vecchio, F., Buffo, P., Sorpresi, F., Frisoni, G. B., et al. (2011). Cortical sources of resting state electroencephalographic rhythms in Parkinson's disease related dementia and Alzheimer's disease. Clin. Neurophysiol. 122, 2355-2364. doi: 10.1016/j.clinph.2011.03.029

Berka, C., Verma, A., Korszen, S., and Johnson, R. (2014). "Development of EEG biomarkers for Alzheimer's disease," in GTC Biomarkers Summit (San Diego, CA).

Bialas, P., and Milanowski, P. (2014). A high frequency steady-state visually evoked potential based brain computer interface using consumer-grade EEG headset. Conf. Proc. IEEE Eng. Med. Biol. Soc. 2014, 5442-5445. doi: 10.1109/EMBC.2014.6944857

Caso, F., Cursi, M., Magnani, G., Fanelli, G., Falautano, M., Comi, G., et al. (2012). Quantitative EEG and LORETA: valuable tools in discerning FTD from AD? Neurobiol. Aging 33, 2343-2356. doi: 10.1016/j.neurobiolaging.2011.12.011

Chen, C. C., Hsu, C. Y., Chiu, H. W., Hu, C. J., and ChiehLee, T. (2015). Frequency power and coherence of electroencephalography are correlated with the severity of Alzheimer's disease: a multicenter analysis in Taiwan. J. Formos Med. Assoc. 114, 729-735. doi: 10.1016/j.jfma.2013.07.008

Garn, H., Waser, M., Deistler, M., Benke, T., Dal-Bianco, P., Ransmayr, G., et al. (2015). Quantitative EEG markers relate to Alzheimer's disease severity in the prospective dementia registry Austria (PRODEM). Clin. Neurophysiol. 126, 505-513. doi: 10.1016/j.clinph.2014.07.005

Goossens, J., Laton, J., Van Schependom, J., Gielen, J., Struyfs, H., Van Mossevelde, S., et al. (2016). EEG dominant frequency peak differentiates between Alzheimer's disease and frontotemporal lobar degeneration. J. Alzheimers Dis. 55, 53-58. doi: 10.3233/JAD-160188

Jakab, A., Kulkas, A., Salpavaara, T., Kauppinen, P., Verho, J., Heikkilä, H., et al. (2014). Novel wireless electroencephalography system with a minimal preparation time for use in emergencies and prehospital care. Biomed. Eng. Online 13:60. doi: 10.1186/1475-925X-13-60

Kim, M., Kim, B. H., and Jo, S. (2015). Quantitative evaluation of a lowcost noninvasive hybrid interface based on EEG and eye movement. IEEE Trans. Neural. Syst. Rehabil. Eng. 23, 159-168. doi: 10.1109/TNSRE.2014.23 65834

Kroupi, E., Castellano, M., Ibanez, D., Montplaisir, J., Gagnon, J. F., Postuma, R., et al. (2017). "Deep networks using auto-encoders for PD prodromal analysis," in Proceedings of 1st HBP Student Conference (Vienna).

Lenartowicz, A., and Loo, S. K. (2014). Use of EEG to diagnose ADHD. Curr. Psychiatry Rep. 16:498. doi: 10.1007/s11920-014-0498-0

Moretti, D. V., Frisoni, G. B., Binetti, G., and Zanetti, O. (2011). Anatomical substrate and scalp EEG markers are correlated in subjects with cognitive impairment and Alzheimer's disease. Front Psychiatry 1:152. doi: $10.3389 /$ fpsyt.2010.00152

Nishida, K., Yoshimura, M., Isotani, T., Yoshida, T., Kitaura, Y., Saito, A., et al. (2011). Differences in quantitative EEG between frontotemporal dementia
Biogen team participated in the study design, analysis, and interpretation of data, in the writing of the report, and in the decision to submit the article for publication. and Alzheimer's disease as revealed by LORET. Clin. Neurophysiol. A 122, 1718-1725. doi: 10.1016/j.clinph.2011.02.011

Pijnenburg, Y. A., Strijers, R. L., Made, Y. V., van der Flier, W. M., Scheltens, P., and Stam, C. J. (2008). Investigation of resting-state EEG functional connectivity in frontotemporal lobar degeneration. Clin. Neurophysiol. 119, 1732-1738. doi: 10.1016/j.clinph.2008.02.024

Ruffini, G., David, I.-S., Castellano, M., and Soria-Frisch, A. (2016). "EEGdriven RNN classification for prognosis of neurodegeneration in at-risk patients," in Internet Corporation for Assigned Names and Numbers (ICANN) (Copenhargen).

Sarnthein, J., and Jeanmonod, D. (2007). High thalamocortical theta coherence in patients with Parkinson's disease. J. Neurosci. 27, 124-131. doi: 10.1523/JNEUROSCI.2411-06.2007

Schiff, S., Casa, M., Di Caro, V., Aprile, D., Spinelli, G., De Rui, M., et al. (2016). A low-cost, user-friendly electroencephalographic recording system for the assessment of hepatic encephalopathy. Hepatology 63, 1651-1659. doi: $10.1002 /$ hep. 28477

Shani Waninger, S. K., Berka, C., Tan, V., and Johnson, R. (2015). "A novel portable neurocognitive biomarker assessment for Parkinson's Disease," in GTC Biomarkers Summit (San Diego, CA)

Snyder, S. M., Rugino, T. A., Hornig, M., and Stein, M. A. (2015). Integration of an EEG biomarker with a clinician's ADHD evaluation. Brain Behav. 5:e00330. doi: $10.1002 / b r b 3.330$

Soria-Frisch, J. M., Ibanez, D., Dunne, S., Grau, C., Ruffini, G., Rodrigues-Brazete, J., et al. (2014). "Machine Learning for a Parkinsons prognosis and diagnosis system based on EEG," in Proceedings International Pharmaco-EEG Society Meeting IPEG (Leipzig).

Taherian, S., Selitskiy, D., Pau, J., and Claire Davies, T. (2017). Are we there yet? Evaluating commercial grade brain-computer interface for control of computer applications by individuals with cerebral palsy. Disabil. Rehabil. Assist. Technol. 12, 165-174. doi: 10.3109/17483107.2015.1111943

Van Hal, B., Rhodes, S., Dunne, B., and Bossemeyer, R. (2014). Low-cost EEGbased sleep detection. Conf. Proc. IEEE Eng. Med. Biol. Soc. 2014, 4571-4574. doi: 10.1109/EMBC.2014.6944641

Waninger, S., Stikic, M., Tatinada, R., and Verma, A. (2016). "Identification of neurophysiological biomarkers of MCI using resting state, EEG", in Alzheimer's Association International Conference (Toronto, ON).

Conflict of Interest Statement: ER and AV: Biogen employee. SW and CB: Advanced Brain Monitoring, Inc employee. GR: Neuroelectrics employee.

Copyright () 2017 Ratti, Waninger, Berka, Ruffini and Verma. This is an open-access article distributed under the terms of the Creative Commons Attribution License (CC $B Y)$. The use, distribution or reproduction in other forums is permitted, provided the original author(s) or licensor are credited and that the original publication in this journal is cited, in accordance with accepted academic practice. No use, distribution or reproduction is permitted which does not comply with these terms. 